\title{
High-Flow Nasal Cannula in Pediatric Patients: A Survey of Clinical Practice
}

\author{
Andrew G Miller RRT RRT-ACCS RRT-NPS, Michael A Gentile MBA RRT FAARC, \\ Lisa M Tyler MHS RRT RRT-NPS, and Natalie Napolitano MPH RRT RRT-NPS FAARC
}

\begin{abstract}
BACKGROUND: High-flow nasal cannula (HFNC) use has greatly increased in recent years. In non-neonatal pediatric patients, there are limited data available to guide HFNC use, and clinical practice may vary significantly. The goal of this study was to evaluate current HFNC practice by surveying practicing pediatric respiratory therapists. METHODS: A survey instrument was posted on the American Association for Respiratory Care's AARConnect online social media platform in March 2017. Paper versions of the survey were also distributed at the annual Children Hospitals Association meeting. RESULTS: There were 63 responses, of which $98 \%$ used HFNC. HFNC was defined as any heated gas delivered by nasal cannula by $49 \%$ of respondents, whereas $21 \%$ defined HFNC as heated gas delivered via nasal cannula at flow greater than or equal to the patient's inspiratory demand, and $16 \%$ defined $\mathrm{HFNC}$ as any gas delivered via nasal cannula above predefined thresholds. Initial flow was set per provider orders by $34 \%$ of respondents, per respiratory therapist-driven protocol by $28 \%$, per patient weight by $15 \%$, per patient age by $15 \%$; $5 \%$ of respondents used other methods. Noninvasive ventilation or CPAP was used by $88 \%$ of respondents as the next step for patients who failed HFNC, with 7\% opting for intubation and $5 \%$ using other interventions. Aerosol therapy was delivered by $75 \%$ of respondents during $\mathrm{HFNC}$, with $77 \%$ of these respondents delivering aerosol via vibrating mesh nebulizer. During aerosol therapy, $13 \%$ of respondents decreased HFNC flow, while $23 \%$ removed patients from HFNC. CONCLUSION: There was no consensus on the definition of HFNC, how to set initial flow, or how to make adjustments. Aerosols were delivered by $75 \%$ of respondents, predominantly via a vibrating mesh nebulizer placed on the dry side of the humidifier. The definition of HFNC, how to set flow, and aerosolized medication delivery are areas in which more research is needed. Key words: high-flow nasal cannula; pediatrics; aerosol therapy; children. [Respir Care 2018;63(7):894-899. () 2018 Daedalus Enterprises]
\end{abstract}

\section{Introduction}

High-flow nasal cannula (HFNC) use has greatly increased in recent years. High-flow gas is defined as a flow that meets or exceeds the patient's inspiratory flow. ${ }^{1}$ Without heated humidity, the amount of gas flow delivered via

\footnotetext{
Mr Miller and Mr Gentile are affiliated with Duke University Medical Center, Durham, North Carolina. Ms Tyler and Ms Napolitano are affiliated with The Children's Hospital of Philadelphia, Philadelphia, Pennsylvania.

The authors have disclosed no conflicts of interest.

Supplementary material related to this paper is available at http:// www.rcjournal.com.
}

nasal cannula is limited due to poor patient tolerance as it dries the nasal mucosa and increases upper-airway resistance. ${ }^{2}$ Technological advances, ease of use, and relative low cost, combined with the widespread availability of commercial systems, has contributed to the increased use of HFNC. HFNC has been shown to have several beneficial physiologic effects, including washout of anatomic

Correspondence: Andrew G Miller RRT RRT-ACCS RRT-NPS, Duke
University Medical Center, Respiratory Care Services, PO Box 3911,
2301, Erwin Rd, Durham, NC 27710. E-mail: andrew.g.miller@duke.edu.

DOI: $10.4187 /$ respcare. 05961 
dead space, more consistent $\mathrm{F}_{\mathrm{IO}_{2}}$ delivery, improved patient tolerance, and low levels of PEEP. ${ }^{3,4}$

A recent systematic review and meta-analysis ${ }^{5}$ in adults found HFNC to be associated with a reduction in intubation rates similar to noninvasive ventilation (NIV) when compared with conventional oxygen therapy, but no changes in ICU length of stay or mortality were reported. The authors concluded that HFNC is an acceptable alternative to NIV in certain clinical situations. A Cochrane review of studies involving premature neonates found HFNC to be equivalent to CPAP regarding mortality, development of chronic lung disease, and extubation failure, although HFNC was associated with less nasal trauma. ${ }^{6}$ However, a more recent randomized controlled trial ${ }^{7}$ found that infants receiving HFNC as primary support were more likely to develop treatment failure within $72 \mathrm{~h}$ when compared to those who received CPAP. A retrospective analysis of a large premature neonatal database found that, compared to CPAP, HFNC was associated with an increased risk of death, bronchopulmonary dysplasia, delayed oral feeding, and increased hospital length of stay for infants $<1 \mathrm{~kg}{ }^{8}$

Randomized controlled trials in pediatric subjects are relatively limited, with a recent Cochrane review being unable to make any recommendations due to no studies meeting their entry criteria. ${ }^{9}$ There has been one randomized controlled trial in infants post cardiac surgery and several in infants with bronchiolitis. ${ }^{10-12}$ Testa et al, ${ }^{10}$ who studied infants post cardiac surgery, found no change in $\mathrm{P}_{\mathrm{aCO}_{2}}$ and an increase in $\mathrm{P}_{\mathrm{aO}}$ when HFNC was compared to low-flow nasal cannula. In infants with bronchiolitis, Milesi et $\mathrm{al}^{11}$ found that HFNC performed worse than CPAP in infants admitted to the pediatric ICU. Kepreotes et $\mathrm{al}^{12}$ found that HFNC resulted in longer time spent on oxygen therapy among infant subjects with mild to moderate bronchiolitis when compared to standard oxygen therapy. Randomized controlled trials in other disease states are currently unavailable.

Due to the limited availability of data to guide HFNC use, we hypothesized that HFNC practice would vary significantly between respondents. In particular, the definition of HFNC, how to set flow, and how to deliver aerosolized medications are areas in which more data are clearly needed. We designed a survey administered to practicing respiratory therapists (RTs) to evaluate current HFNC practices in pediatric patients.

\section{Methods}

After a literature review, we designed a survey instrument using REDCap. The survey instrument was then sent to 5 colleagues from different centers with experience in HFNC use and survey research for feedback. Changes were made based on their feedback, and the survey was

\section{QUICK LOOK}

\section{Current knowledge}

High-flow nasal cannula (HFNC) use has greatly increased in recent years. In non-neonatal pediatric patients, limited data are available to guide HFNC use, and clinical practice may vary significantly.

\section{What this paper contributes to our knowledge}

There was no consensus on the definition of HFNC, how to set initial flow, or how to make adjustments. Failure of HFNC resulted in escalation to noninvasive ventilation or CPAP for $88 \%$ of respondents. Aerosols were delivered by $75 \%$ of respondents, predominantly via a vibrating mesh nebulizer placed on the dry side of the humidifier.

then posted on the neonatal/pediatric, management, and help line sections of the American Association for Respiratory Care (AARC) AARConnect online social media platform in March 2017. Paper versions of the survey were distributed at the annual Children Hospitals Association (CHA) meeting. Paper surveys were entered into REDCap by one of the authors. The survey was approved by the AARC board of directors for distribution via AARConnect and approved for exemption by the Children's Hospital of Philadelphia's Institutional Review Board. Responses from the AARConnect and CHA were analyzed for differences in clinical practice and are summarized in supplementary materials (see the supplementary materials at http://www.rcjournal.com). More than one respondent was allowed per institution.

The survey included questions about hospital and respondent demographics, initial HFNC flow setting, adjustments, locations where HFNC is used, frequency of assessments, parameters evaluated during assessments, and aerosol delivery via HFNC if used (see the supplementary materials at http://www.rcjournal.com). Data were analyzed using SPSS version 24 (IBM, Chicago, Illinois). Chi-square tests and unpaired $t$ tests were performed for categorical and continuous data, respectively.

\section{Results}

There were 36 responses from AARConnect and 27 paper surveys from the CHA; $98 \%$ of respondents indicated use of HFNC. Respondent demographics are summarized in Table 1. There were no respondents with identical demographic data. CHA respondents were more likely to be from standalone children's hospitals compared to respondents on AARConnect (100\% vs $28 \%$, $P<.001)$ or to be a manager/director $(89 \%$ vs $44 \%$, 
Table 1. Respondent Demographics

\begin{tabular}{|c|c|c|c|c|}
\hline Demographics & AARConnect & Children's Hospital Association & Total & $P$ \\
\hline Respondents, $n$ & 36 & 27 & 63 & \\
\hline Number of beds, mean \pm SD & $84 \pm 91$ & $346 \pm 127$ & $198 \pm 169$ & $<.001$ \\
\hline Number of PICU beds, mean \pm SD & $21 \pm 26$ & $130 \pm 121$ & $66 \pm 96$ & $<.001$ \\
\hline Standalone children's hospital, $n(\%)$ & $10(28)$ & $27(100)$ & $37(59)$ & $<.001$ \\
\hline Tertiary/academic center, $n(\%)$ & $23(64)$ & $22(81)$ & $45(71)$ & .10 \\
\hline \multicolumn{5}{|l|}{ Role, $n(\%)$} \\
\hline Manager/director & $16(44)$ & $24(89)$ & $40(63)$ & .001 \\
\hline Supervisor/clinical specialist & $9(25)$ & $1(4)$ & $10(16)$ & \\
\hline Other & $2(13)$ & $2(7)$ & $4(6)$ & \\
\hline Staff therapist & $9(25)$ & $0(0)$ & $9(14)$ & \\
\hline Use HFNC, $n(\%)$ & $35(97)$ & $27(100)$ & $62(98)$ & .38 \\
\hline Neonates & $31(85)$ & $27(100)$ & $58(86)$ & .044 \\
\hline Pediatrics & $32(89)$ & $27(100)$ & $59(94)$ & .07 \\
\hline Adults & $28(78)$ & $12(44)$ & $40(63)$ & .007 \\
\hline \multicolumn{5}{|l|}{ Where HFNC is used, $n(\%)$} \\
\hline Emergency department & $29(81)$ & $26(96)$ & $55(87)$ & .063 \\
\hline Regular floors & $21(58)$ & $20(74)$ & $41(65)$ & .20 \\
\hline Step-down or intermediate care & $27(75)$ & $19(70)$ & $46(73)$ & .68 \\
\hline ICU & $33(92)$ & $27(100)$ & $60(95)$ & .12 \\
\hline $\begin{array}{l}\text { HFNC }=\text { high-flow nasal cannula } \\
\text { PICU }=\text { pediatric intensive care unit }\end{array}$ & & & & \\
\hline
\end{tabular}

$P<.001)$. CHA respondents were more likely than AARConnect respondents to use HFNC in neonates $(100 \%$ vs $85 \%, P=.044)$, and less likely to use HFNC in adults $(44 \%$ vs $78 \%, P=.007)$. There were no statistically significant differences in location of HFNC use. There were no statistically significant differences in clinical practice between the AARC and CHA respondents. Individual differences are included in the online supplemental materials (see the supplementary materials at http://www.rcjournal.com).

Disease states, parameters monitored during RT assessments, and frequency of assessments based on patient location are summarized in Table 2. Data on the definition of HFNC, initial HFNC setting, adjustments, and what is generally done when the patient is failing HFNC are summarized in Table 3.

Aerosol therapy was delivered by $75 \%$ of respondents during HFNC. Of these, $77 \%$ delivered aerosols via a vibrating mesh nebulizer, $6 \%$ via a jet nebulizer, $13 \%$ decreased HFNC flow, and $23 \%$ took the patient off HFNC to deliver treatment with a metered-dose inhaler or jet nebulizer. Medications delivered via aerosol therapy included $\beta_{2}$ agonists for $92 \%$ of respondents, corticosteroids for $50 \%$, mucolytics for $44 \%$, other medications for $4 \%$, and one respondent indicated they delivered all respiratory medications, including epoprostenol, via aerosol therapy. The nebulizer was placed on the dry side of the humidifier by $60 \%$ of respondents, on the wet side of the humidifier by $6 \%$, and between the circuit and the cannula by $17 \%$.

\section{Discussion}

In this survey of RTs, there was no consensus for the definition of HFNC in pediatric patients, or how to set or adjust flow. Most respondents used CPAP or NIV when patients failed HFNC. Most respondents indicated that RTs assessed patients every $4 \mathrm{~h}$. More than three quarters of respondents delivered aerosol therapy via HFNC, and most used a vibrating mesh nebulizer placed on the dry side of the humidifier.

HFNC was defined as any heated gas delivered by nasal cannula for $49 \%$ of respondents, while $21 \%$ indicated they set the flow at or above the patient's inspiratory flow. Importantly, no respondents indicated that they used a scientifically validated method to ensure set flow was greater than or equal to the patient's inspiratory flow. Walsh and Smallwood ${ }^{13}$ have put forth a method to approximate the initial flow setting for HFNC based on the patient's age and weight; to our knowledge, however, this method has not been validated in clinical practice.

Clearly defining HFNC is important because a standard definition for HFNC would allow clinicians to compare patient outcomes across centers, to provide standard definitions for clinical trials, and to provide program benchmarking. The use of a clear definition of HFNC has important patient safety 


\section{HFNC Use by RTs Caring for Pediatric Patients}

Table 2. Patient Characteristics and Frequency of RT Assessments

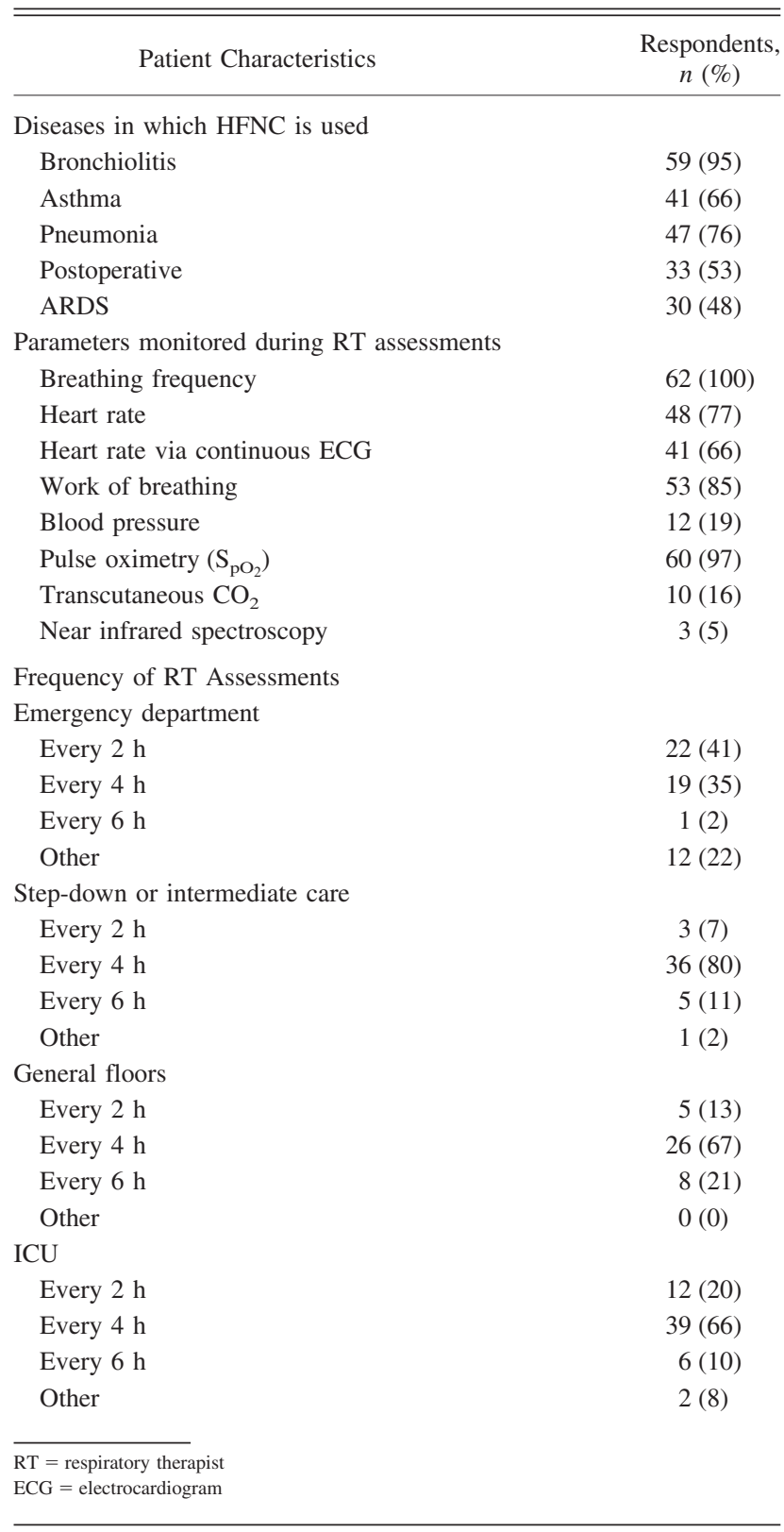

implications as well, because many centers require patients on HFNC to be in the ICU environment. Determining which patient populations, set flows, and $\mathrm{F}_{\mathrm{IO}_{2}}$ requirements are safe to be outside the ICU are important areas where additional data are needed. Therapeutic goals also need to be taken into consideration, as patients placed on HFNC to receive aerosol therapy or therapeutic gases, or decrease discomfort associated with regular nasal cannula use, may not require ICUlevel care. The frequency of monitoring and assessments by RTs could be determined on the basis of therapeutic goals to optimize therapy and to avoid unnecessary assessments. These parameters will likely need to be determined according to the
Table 3. Respondent Survey Responses

\begin{tabular}{|c|c|}
\hline Survey Responses & $n(\%)$ \\
\hline \multicolumn{2}{|l|}{ A. How do you define HFNC? } \\
\hline $\begin{array}{l}\text { Any heated/humidified and blended gas } \\
\text { delivered via nasal cannula }\end{array}$ & $31(49)$ \\
\hline $\begin{array}{l}\text { Heated gas delivered via nasal cannula at flow } \\
\text { greater or equal to the patient's inspiratory } \\
\text { demand }\end{array}$ & $13(21)$ \\
\hline $\begin{array}{l}\text { Nasal cannula flow above predefined thresholds } \\
\text { based on the patient's age or weight }\end{array}$ & $10(16)$ \\
\hline Other/no response & $6(10)$ \\
\hline \multicolumn{2}{|l|}{ B. What method is used to set flow? } \\
\hline Per provider orders & $21(34)$ \\
\hline Per RT-driven protocol & $17(28)$ \\
\hline Weight-based (eg, 1 L/kg/min) & $9(15)$ \\
\hline Age based (different flow for different ages) & $9(15)$ \\
\hline Other & $3(5)$ \\
\hline \multicolumn{2}{|l|}{ C. How is flow adjusted? } \\
\hline Per RT-driven protocol & $22(36)$ \\
\hline $\begin{array}{l}\text { On the basis of vital signs (breathing frequency, } \\
\text { heart rate, work of breathing) }\end{array}$ & $21(34)$ \\
\hline Per provider orders & $15(25)$ \\
\hline Other & $3(5)$ \\
\hline \multicolumn{2}{|l|}{$\begin{array}{l}\text { D. In general, when a patient's oxygenation on } \\
\text { HFNC is below goal, what do you do first? }\end{array}$} \\
\hline Increase $\mathrm{F}_{\mathrm{IO}_{2}}$ & $34(57)$ \\
\hline Increase flow & $18(30)$ \\
\hline Other & $8(13)$ \\
\hline \multicolumn{2}{|l|}{$\begin{array}{l}\text { E. In general, what is the next step if a patient } \\
\text { fails HFNC? }\end{array}$} \\
\hline Noninvasive ventilation & $37(63)$ \\
\hline CPAP & $15(25)$ \\
\hline Intubation & $4(7)$ \\
\hline Other & $3(5)$ \\
\hline \multicolumn{2}{|c|}{$\begin{array}{l}\text { For A, } N=62 ; \text { for } \mathrm{B}, N=61 \text {; for } \mathrm{C}, N=61 ; \text { for } \mathrm{D}, N=60 \text {; for } \mathrm{E}, N=59 . \\
\mathrm{HFNC}=\text { high-flow nasal cannula } \\
\mathrm{RT}=\text { respiratory therapist }\end{array}$} \\
\hline
\end{tabular}

needs of individual institutions and their comfort level utilizing HFNC in areas with less frequent monitoring than the ICU or the emergency department.

Optimal methods to determine initial flow and to adjust the flow for HFNC are unknown. Initial flows in clinical trials have used $2 \mathrm{~L} / \mathrm{kg} / \mathrm{min}$ in infants post cardiac surgery and in infants with moderate/severe bronchiolitis or pneumonia, $1 \mathrm{~L} / \mathrm{kg} / \mathrm{min}$ for infants with mild/moderate bronchiolitis, and $1-3 \mathrm{~L} / \mathrm{kg} / \mathrm{min}$ for subjects in the pediatric ICU with respiratory distress due to bronchiolitis and pneumonia. ${ }^{10-12,14,15}$ Weiler et al ${ }^{16}$ recently evaluated HFNC titration in 21 children (median age 6 mo, median weight $6.5 \mathrm{~kg}$ ) and found the optimal flow to decrease work of breathing (as measured by the pressure rate product) was $1.5-2 \mathrm{~L} / \mathrm{kg} / \mathrm{min}$. Importantly, the authors did not increase flow above $2 \mathrm{~L} / \mathrm{kg} / \mathrm{min}$ due to concerns about air leak and subject tolerance. ${ }^{16}$ Only $19 \%$ of our respondents indi- 


\section{HFNC Use by RTs Caring for Pediatric Patients}

cated that a weight-based method was used to set initial inspiratory flow. The most common response was per provider orders; we did not ask specifically what methods were used by the providers to determine initial flow. Conceptually, using patient weight seems reasonable, but older children may not tolerate flows of $2 \mathrm{~L} / \mathrm{kg} / \mathrm{min}$. In addition, some children may be significantly under or over their predicted body weight, thus making flow based on weight suboptimal. Using age instead of weight is another strategy, but many patients are underweight or overweight based on their age, especially children with chronic respiratory illnesses who tend to be below their predicted body weight. ${ }^{17}$ Direct comparisons of different methods for setting and adjusting flow are needed. Individual inspiratory flows also likely vary substantially between disease states, even for patients who are the same age, height, and weight. Device limitations prevent delivering $>2 \mathrm{~L} / \mathrm{kg} / \mathrm{min}$ for larger children because most devices have a maximum flow of $60 \mathrm{~L} / \mathrm{min}$.

Most of our respondents indicated that NIV or CPAP was the next intervention for patients who did not respond well to HFNC. We did not ask respondents what criteria were used to determine HFNC failure; however, it is important to recognize when HFNC is failing and escalate to appropriate level of support. When starting HFNC, clear clinical goals and criteria for escalation should be defined by the team, as well as whether the next step will be NIV, CPAP, or intubation. A delay in escalation to NIV may be associated with worse outcomes in children with asthma. ${ }^{18}$

Aerosol delivery via HFNC is somewhat controversial. ${ }^{19,20}$ Bench studies to date indicate that aerosol is delivered to the airway; however in vivo data from humans are lacking. Aerosol delivery is also affected by flow, type of system used, cannula size, and type of nebulizer used. ${ }^{21}$ The majority of our respondents used vibrating mesh nebulizers to deliver aerosol therapy to patients, a method that has several advantages compared to jet nebulizers as it does not add flow to the system and does not require removal from the system for therapy. The optimal delivery method is also affected by the type of commercially available HFNC set-up used by individual centers. Differences between the CHA respondents and AARConnect respondents may have been due to the system(s) used at each center; we did not ask respondents what system(s) they used. Future research should focus on in vivo studies of not only aerosol deposition, but patient-oriented outcomes such as length of stay for asthmatic patients or hospital admission rate for those in the emergency department.

This study has several limitations. The relatively small sample size and demographic differences between the AARConnect respondents and CHA may have biased the results. Respondents may have had special interest in HFNC practice. There were no statistically significant differences between the groups for clinical practice; however, the sur- vey was underpowered to detect differences. In an effort to keep the survey brief, subtleties in clinical practice could not be detected. Only members of the CHA and AARC members with access to the AARConnect Neonatal/Pediatric, Management, and Help Line sections were surveyed, and these respondents may not be representative of RTs as a whole. Questions may not have been worded clearly, and by their nature surveys cannot evaluate nuances in clinical practice. Finally, to keep the survey focused, we did not ask any questions regarding HFNC weaning methods or strategies. These topics are also debated and are deserving of further study.

\section{Conclusion}

There was no consensus on the definition of HFNC, how to set initial flow, or how to make adjustments. Failure of HFNC resulted in escalation to NIV or CPAP for $88 \%$ of respondents. Aerosols were delivered by $75 \%$ of respondents, predominantly via a vibrating mesh nebulizer placed on the dry side of the humidifier. The definition of HFNC, how to set flow, and aerosolized medication delivery are areas in which more research is needed.

\section{REFERENCES}

1. Hess D. Respiratory care: principles and practice. Sudbury, MA: Jones \& Bartlett Learning; 2012:285.

2. Williams R, Rankin N, Smith T, Galler D, Seakins P. Relationship between the humidity and temperature of inspired gas and the function of the airway mucosa. Crit Care Med 1996;24(11):1920-1929.

3. Lee JH, Rehder KJ, Williford L, Cheifetz IM, Turner DA. Use of high-flow nasal cannula in critically ill infants, children, and adults: a critical review of the literature. Intensive Care Med 2013;39(2): 247-257.

4. Ward JJ. High-flow oxygen administration by nasal cannula for adult and perinatal patients. Respir Care 2013;58(1):98-122.

5. Ni YN, Luo J, Yu H, Liu D, Ni Z, Cheng J, et al. Can high-flow nasal cannula reduce the rate of endotracheal intubation in adult patients with acute respiratory failure compared with conventional oxygen therapy and noninvasive positive pressure ventilation? A systematic review and meta-analysis. Chest 2017;151(4):764-775.

6. Wilkinson D, Andersen C, O'Donnell CP, De Paoli AG, Manley BJ. High-flow nasal cannula for respiratory support in preterm infants. Cochrane Database Syst Rev 2016;2:CD006405.

7. Roberts CT, Owen LS, Manley BJ, Froisland DH, Donath SM, Dalziel KM, et al. Nasal high-flow therapy for primary respiratory support in preterm infants. N Engl J Med 2016;375(12):1142-1151.

8. Taha DK, Kornhauser M, Greenspan JS, Dysart KC, Aghai ZH. High-flow nasal cannula use is associated with increased morbidity and length of hospitalization in extremely low birth weight infants. J Pediatr 2016;173:50-55.

9. Mayfield S, Jauncey-Cooke J, Hough JL, Schibler A, Gibbons K, Bogossian F. High-flow nasal cannula therapy for respiratory support in children. Cochrane Database Syst Rev 2014(3):CD009850.

10. Testa G, Iodice F, Ricci Z, Vitale V, De Razza F, Haiberger R, et al. Comparative evaluation of high-flow nasal cannula and conventional oxygen therapy in paediatric cardiac surgical patients: a randomized controlled trial. Interact Cardiovasc Thorac Surg 2014;19(3):456-461. 


\section{HFNC Use by RTs Caring for Pediatric Patients}

11. Milesi C, Essouri S, Pouyau R, Liet JM, Afanetti M, Portefaix A, et al. High-flow nasal cannula (HFNC) versus nasal continuous positive airway pressure (nCPAP) for the initial respiratory management of acute viral bronchiolitis in young infants: a multicenter randomized controlled trial (TRAMONTANE study). Intensive Care Med 2017;43(2):209-216.

12. Kepreotes E, Whitehead B, Attia J, Oldmeadow C, Collison A, Searles A, et al. High-flow warm humidified oxygen versus standard low-flow nasal cannula oxygen for moderate bronchiolitis (HFWHO RCT): an open, phase 4, randomised controlled trial. Lancet 2017; 389(10072):930-939.

13. Walsh BK, Smallwood CD. Pediatric oxygen therapy: a review and update. Respir Care 2017;62(6):645-661.

14. Chisti MJ, Salam MA, Smith JH, Ahmed T, Pietroni MA, Shahunja $\mathrm{KM}$, et al. Bubble continuous positive airway pressure for children with severe pneumonia and hypoxaemia in Bangladesh: an open, randomised controlled trial. Lancet 2015;386(9998):1057-1065.

15. Vitaliti G, Vitaliti MC, Finocchiaro MC, Di Stefano VA, Pavone P, Matin N, et al. Randomized comparison of helmet CPAP versus high-flow nasal cannula oxygen in pediatric respiratory distress. Respir Care 2017;62(8):1036-1042.

16. Weiler T, Kamerkar A, Hotz J, Ross PA, Newth CJL, Khemani RG. The relationship between high-flow nasal cannula flow rate and effort of breathing in children. J Pediatr 2017;189:66-71.

17. Proesmans M. Respiratory illness in children with disability: a serious problem? Breathe (Sheff) 2016;12(4):e97-e103.

18. Pilar J, Modesto IAV, Lopez-Fernandez YM, Lopez-Macias O, Garcia-Urabayen D, Amores-Hernandez I. High-flow nasal cannula therapy versus non-invasive ventilation in children with severe acute asthma exacerbation: an observational cohort study. Med Intensiva 2017;41(7):418-424.

19. DiBlasi RM. Clinical controversies in aerosol therapy for infants and children. Respir Care 2015;60(6):894-914.

20. Hess DR. Aerosol therapy during noninvasive ventilation or highflow nasal cannula. Respir Care 2015;60(6):880-891.

21. Berlinski A. Pediatric aerosol therapy. Respir Care 2017;62(6):662677. 